\title{
MODELAGEM E SIMULAÇÃO DE EVENTOS DISCRETOS COMO FERRAMENTA MEDIADORA DA APRENDIZAGEM BASEADA EM PROBLEMAS
}

\author{
MODELING AND SIMULATION OF DISCRETE EVENTS AS A MEDIATING TOOL FOR \\ PROBLEM-BASED LEARNING
}

Jackson Roberto Eleotério ${ }^{1}$, Simone Leal Schwertl ${ }^{2}$

DOI: 10.37702/REE2236-0158.v40p279-287.2021

\begin{abstract}
RESUMO
As metodologias ativas de aprendizagem incontestavelmente devem compor o projeto pedagógico dos cursos de engenharia, pois contribuem para o alcance das habilidades e competências necessárias para o exercício profissional pleno. A Aprendizagem Baseada em Problemas (Problem-Based Learning - PBL) é uma das metodologias mais recomendadas. Entretanto, como propor problemas concretos vinculados aos processos industriais, que envolvam abordagens interdisciplinares, próximos da realidade profissional do estudante e que possam ser manipulados durante a busca por soluções? O objetivo deste trabalho é apresentar uma proposta de uso de modelagem e simulação de eventos discretos como ferramenta mediadora da Aprendizagem Baseada em Problemas. Os passos de formulação do problema, modelagem conceitual, modelagem computacional e utilização do modelo na busca por soluções para o problema proposto são detalhados. Um exemplo de adoção dessa ferramenta nas disciplinas vinculadas à industrialização da madeira é relatado, com destaque para a associação com as atividades de pesquisa e extensão.
\end{abstract}

Palavras-chave: processos industriais; modelagem conceitual; modelagem computacional; simulação estocástica; ensino assistido por computador.

\begin{abstract}
Active learning methodologies must undoubtedly be part of the pedagogical project of engineering courses, as they contribute to achieving the skills and competencies necessary for full professional practice. Problem-Based Learning (PBL) is one of the most recommended methodologies. However, how to propose concrete problems linked to industrial processes, which involve interdisciplinary approaches, close to the student's professional reality and that can be manipulated during the search for solutions? The objective of this work is to present a proposal for the use of modeling and simulation of discrete events as a mediating tool for Problem-Based Learning. The steps of formulating the problem, conceptual modeling, computational modeling and using the model in the search for solutions to the proposed problem are detailed. An example of the adoption of this tool in disciplines related to the industrialization of wood is reported, with emphasis on the association with research and extension activities.
\end{abstract}

Keywords: industrial processes; conceptual modeling; computational modeling; stochastic simulation; computer-assisted teaching.

\footnotetext{
${ }^{1}$ Professor Dr. em Engenharia Química, Universidade Regional de Blumenau (FURB); eleoterio@furb.br

${ }^{2}$ Professora Dra. em Educação Científica e Tecnológica, Universidade Regional de Blumenau (FURB); sileal@furb.br
} 


\section{INTRODUÇÃO}

Os processos de industrialização são complexos, pois envolvem inter-relações entre a matéria-prima, as máquinas, os métodos, as condições ambientais, os recursos humanos e o mercado.

A experiência tem demonstrado que a formação de engenheiros preparados para analisar, atuar e padronizar processos industriais é dificultada pela reduzida capacidade de abstração dos alunos, especialmente os que não têm vivência prévia em ambientes industriais. Uma alternativa para amenizar essa situação é a substituição parcial do aprendizado através da audiência de aulas expositivas pela Aprendizagem Baseada em Problemas (Problem-Based Learning - PBL).

A implantação de metodologias ativas ${ }^{3}$ de aprendizagem tem resultado em aumento no nível de satisfação dos alunos, no melhor entendimento das inter-relações entre disciplinas e no encorajamento dos estudantes para tomarem para si a responsabilidade pelo seu aprendizado (LÓPEZ-QUEROL et al., 2015). Mas, como propor problemas reais relacionados aos processos de industrialização de maneira que possam ser manipulados pelos alunos e que direcionem o aprendizado?

Entre os objetivos deste trabalho estão: apresentar uma proposta de uso da modelagem e simulação de eventos discretos associada à PBL como uma ferramenta de aprendizagem ativa do ensino de processos industriais, compartilhar a experiência da sua implantação ao longo de cinco anos na temática de industrialização da madeira e validar os resultados junto à comunidade científica.

\section{MODELAGEM E SIMULAÇÃO DE EVENTOS DISCRETOS COMO FERRAMENTA PARA ABORDAR PROBLEMAS MAIS PRÓXIMOS DA REALIDADE DO FUTURO ENGENHEIRO}

\footnotetext{
${ }^{3}$ Entendemos as metodologias ativas de aprendizagem como um conjunto de estratégias que permitam uma educação que pressuponha a atividade, ao contrário da passividade, por parte dos estudantes, em que o aluno se torna protagonista e assume mais responsabilidade sobre seu
}

No que se refere à modelagem e à simulação, cabe apontar que um sistema de produção pode ser analisado a partir de sua constituição real, entretanto os modelos abrem outras possibilidades, tais como: comprimir ou expandir o tempo de observação, controlar as causas de variação, ter a possibilidade de interromper o processo em questão e revisá-lo, ter a possibilidade de restaurar uma determinada condição do sistema e realizar replicações (HERNÁNDEZ-DE-MENÉNDEZ et al., 2019, CWIF; MEDINA, 2015).

A taxonomia dos modelos apresentada por Babulak e Wang (2010) permite dividi-los em modelos físicos e computacionais. Nos primeiros, os sistemas são representações físicas, normalmente em escala reduzida, do sistema, como uma maquete. Os modelos computacionais são preferidos pela capacidade de se alterar parâmetros do sistema mais rapidamente, além de permitir a coleta de um maior número de informações em menor intervalo de tempo. Ainda, os modelos computacionais podem ser divididos em determinísticos e estocásticos. Os modelos estocásticos permitem que sejam consideradas as variações aleatórias observadas nos parâmetros do sistema real. Um inconveniente desse grupo de modelos é a necessidade de se coletar um maior número de dados para modelar as distribuições de probabilidade envolvidas. Em relação à mudança ao longo do tempo, os modelos são classificados em estáticos ou dinâmicos. Os últimos permitem a avaliação do desempenho do sistema ao longo do tempo. Entre os sistemas dinâmicos, há uma predominância por aqueles que avaliam o estado do sistema em momentos discretos do tempo. Os modelos computacionais estocásticos dinâmicos discretos permitem a manipulação de muitos problemas reais propostos na PBL.

Os modelos de eventos discretos, implantados em softwares específicos para esse propósito, são representações de um processo real com objetivo de responder a questões específicas envolvendo as condições atuais do

processo de aprendizagem. Com esse entendimento, o papel do professor como guia e mediador da aprendizagem, colocando-se ao lado do estudante, é fortalecido e é cada vez mais importante (BACICH; MORAN, 2018). 
sistema ou cenários hipotéticos. Os parâmetros que alimentam os modelos partem da observação do desempenho de sistemas reais nos aspectos de tempos de processamento, intervalos entre entregas, frequência e duração de interrupções no processo, entre outros (CWIF; MEDINA, 2015). Os dados reais são modelados por distribuições de probabilidade e assim as aleatoriedades são consideradas (SPEDDING; SUN, 1999). A aplicação do estudo das probabilidades na análise de sistemas reais modelados e simulados como eventos discretos é apontada por Romeu (1997) como um recurso para motivar os alunos a aprenderem estatística. A modelagem e a simulação são apontadas ainda pelo mesmo autor como uma alternativa à demorada e dispendiosa experimentação em sistemas reais.

Mesquita, Mariz e Tomotani (2017) apresentam, como ferramenta de ensino, a experiência da modelagem de unidades fabris por meio de simulação de eventos discretos. Os resultados apontaram aumento na motivação e consolidação do aprendizado.

\section{UMA PROPOSTA DE APRENDIZAGEM BASEADA EM PROBLEMAS MEDIADA POR MODELAGEM E SIMULAÇÃO DE EVENTOS DISCRETOS}

A formação de engenheiros capazes de analisar sistemas industriais de produção pode ser beneficiada pelo desenvolvimento de uma metodologia que possibilite a obtenção da solução de problemas mediada por modelagem e simulação de eventos discretos. Ou seja, uma metodologia que contemple tanto os passos recomendados para a PBL (ELMÔR FILHO et al., 2019) quanto para modelagem e simulação (CHWIF; MEDINA, 2015; ROBINSON, 2008a)

Entre os elementos essenciais que devem estar presentes no PBL estão: o estabelecimento do problema, coerente com a área de atuação profissional dos alunos, apresentado anteriormente à teoria; o estabelecimento de um processo formal de solução de problemas; e o trabalho em grupos de alunos que estudam o assunto de maneira autônoma, integrando conhecimentos (RIBEIRO, 2008).

Nesse contexto, a modelagem pode ser vista como uma forma de transcrever o problema e a simulação como uma forma de obtenção de soluções.

A seguir são apresentados os passos da metodologia de aprendizagem ativa intitulada Solução de Problemas mediada por Modelagem e Simulação (SPMMS), construída com base na sinergia das orientações da PBL e da modelagem e simulação.

\section{Formulação do problema e nivelamento conceitual}

A formulação clara de um problema real vinculado a um processo industrial é a etapa que marca o início da Aprendizagem Baseada em Problemas. Processos em que os alunos tenham familiaridade ou que permitam a visualização e o acompanhamento da operação em um sistema real são os que apresentam maior chance de engajamento.

Depois de estabelecido o problema é importante o estabelecimento de um referencial teórico em que as principais etapas sejam detalhadas e as possíveis influências de fatores relacionados às máquinas, matéria-prima, métodos, medidas, meio ambiente e mão de obra sejam identificadas.

Essa etapa termina com a concepção de uma ou mais questões relevantes relacionadas ao problema, que deverão ser respondidas pelos alunos com base nos resultados do modelo de simulação. Propor soluções a essas questões justifica a atividade, pois a simulação não se justifica apenas pela própria simulação (CHWIF; MEDINA, 2015).

\section{Delimitação e estabelecimento do modelo conceitual}

Por modelagem conceitual se entende um processo de abstração da realidade que parte do problema estabelecido para definir o que vai ser modelado, quais os dados necessários e quais as suposições, as simplificações, os pressupostos e as hipóteses simplificadoras. Nessa etapa é que 
as variáveis respostas às questões estabelecidas são definidas e a sua importância é fundamental na validade do modelo e na confiabilidade dos resultados (ROBINSON, 2008a). Essa etapa corresponde à geração de hipóteses e à identificação de fatores relevantes (ELMOR FILHO et al, 2019).

Não há um único modelo conceitual correto, Robinson (2008b) inclusive considera uma arte a definição de um modelo conceitual, pois não há uma regra para se definir o grau de simplificação e os pressupostos assumidos no modelo. É certo que esse modelo deve ser simples o suficiente para responder as questões propostas, pois modelos simples requerem menos tempo de desenvolvimento e de coleta de dados (ROBINSON, 2020).

No roteiro de aplicação proposto por Elmôr Filho et al. (2019) esses passos correspondem à confrontação das hipóteses com os dados disponíveis e às primeiras tentativas de simulação. Não havendo sucesso, as equipes definem o que e como realizarão a coleta de informações adicionais.

A última etapa da modelagem conceitual é a validação, momento em que a capacidade do modelo em responder as questões propostas que justificam sua criação é avaliada. Um modelo válido é aquele que tem potencial para atender aos objetivos do projeto. Ao longo do processo de modelagem, a validação pode ser uma etapa recorrente, em que ajustes são feitos no modelo computacional, inclusive com a coleta de novos dados. Uma ênfase na formação de estudantes, proporcionando uma sólida apropriação da modelagem conceitual, é apontada por Zee et al. (2010) como um passo importante na qualificação dos modelos de simulação.

\section{Estabelecimento \\ do modelo computacional}

O modelo computacional é a representação do modelo conceitual em um software ou linguagem de simulação. As variabilidades observadas nos tempos de processamento, de movimentação e nas características ou atributos dos elementos ou entidades em processamento e as decisões que devem ser tomadas no sistema real são representadas no software escolhido. A dificuldade dessa etapa se limita ao conhecimento do usuário sobre o software (CWIF; MEDINA, 2015).

Bons resultados têm sido obtidos quando se aumenta paulatinamente o grau de complexidade do modelo computacional, primeiro adotando valores determinísticos e depois estocásticos, além de se verificar frequentemente a sua funcionalidade (MESQUITA; MARIZ; TOMOTANI, 2017).

$\mathrm{Na}$ etapa voltada mais diretamente ao ensino dos objetos de conhecimento, o aluno já deverá dominar o entendimento do sistema e passará a observar a sua representação gráfica segundo uma perspectiva dinâmica. Esse passo corresponde ao que Elmôr Filho et al. (2019) estabelecem como a aplicação do conhecimento construído na resolução do problema até que a equipe alcance uma solução satisfatória.

Finalizada a verificação do modelo computacional este pode ser utilizado para observar a relação entre variáveis de entrada e variáveis resposta, caracterizando-se a fase de experimentação. Nessa fase os objetivos definidos no início do processo de simulação são recuperados e o modelo é utilizado para a obtenção de respostas. No paralelo estabelecido com as etapas propostas por Elmôr Filho (2019) está a etapa correspondente à retomada ou aprofundamento de algumas questões e a produção de um relatório que representa a resolução final do problema.

\section{EXEMPLO DE APLICAÇÃO NAS DISCIPLINAS DE PROCESSOS DE INDUSTRIALIZAÇÃO DA MADEIRA}

\section{A motivação: ambiente externo e mudanças no perfil dos alunos}

Como todo setor industrial, o de industrialização da madeira passa por mudanças rápidas e profundas em resposta à pressão por aumento da eficiência, redução das margens de lucro, competição em escala globalizada, redução na disponibilidade de mão de obra e a frequente entrada no mercado de competidores (TAO et al., 2019). 
O reflexo no ensino tem sido a redução na importância do estudo das propriedades da madeira e o aumento sobre os seus processos de industrialização, como pode ser exemplificado pelas alterações do Projeto Pedagógico de curso de Engenharia Florestal (FURB, 2010). Se antes as unidades de processamento da madeira eram analisadas pelo seu layout, pelas características de cada uma das possíveis máquinas, agora há necessidade de se avaliar a dinâmica do sistema.

A forma com que engenheiros são preparados para atuar nos processos de industrialização da madeira também mudou. Passou de uma visão determinística do processo para uma abordagem em que as inter-relações entre os fatores internos e externos da indústria devem ser consideradas, o que Strimel, Grubbs e Wells (2017) chamam de systems thinking.

A convicção para a adoção da modelagem e simulação em sinergia com os pressupostos da PBL é complementada pelo fato de as relações que afetam uma unidade de processamento de madeira serem complexas, dinâmicas e a experimentação ou o isolamento de fatores em um sistema real é praticamente impossível.

De outra parte, mudanças no perfil das turmas dos cursos de engenharia - como a redução do número de alunos e o aumento das opções de cursos e de instituições - também estimulam a adoção de metodologias que conduzam a aprendizagem ativa, na perspectiva de melhorar a qualidade do processo ensinoaprendizagem e aproximar os estudantes da realidade da futura profissão.

No que tange ao perfil dos estudantes não menos importante são as características dos nativos digitais, os quais contemplam outras linguagens, visuais e sonoras (SANTAELLA, 2013). Portanto, estes se beneficiam da utilização de componentes visuais, como os utilizados nos modelos de simulação de eventos discretos, para entender a representação de problemas mais complexos do ambiente industrial. As características de distintas gerações são apontadas por Moore, Jones e Frazier (2017) como aspectos que devem ser considerados no processo de formar profissionais capazes de entender e descrever um problema e suas delimitações, desenvolver e testar soluções potenciais e entregar um produto final que é bem definido e que atenda às demandas dos usuários.

A aplicação da metodologia SPMMS no ensino de processos de industrialização da madeira tem como horizonte complementar a formação de competências e habilidades previstas nas Diretrizes Curriculares Nacionais para os cursos de graduação em Engenharia Florestal, em especial: conhecer e compreender os fatores de produção e combiná-los com eficiência técnica e econômica; conceber, projetar e analisar sistemas, produtos e processos; identificar problemas e propor soluções; desenvolver e utilizar novas tecnologias; gerenciar, operar e manter sistemas e processos e atuar com espírito empreendedor (CNE/CES, 2006; FURB, 2010).

\section{Uma aplicação da SPMMS a um processo de industrialização da madeira}

Normalmente a aplicação da metodologia inicia com a modelagem do problema. No exemplo adotado, esse processo começa com a observação de uma planta baixa ou layout do sistema que está sendo analisado.

Apesar do layout contribuir para o entendimento do sistema, ele não menciona as decisões e os tempos de operação. A conversão de um layout em um fluxograma agrega o estabelecimento das decisões durante cada operação. Com a elaboração de um fluxograma será possível observar que decisões devem ser tomadas ao longo do processo e, ainda, que essas demandam de dados.

Compreendido o processo como algo dinâmico, uma pergunta que norteará a solução do problema é formulada. Por exemplo: qual a influência do diâmetro das toras na produtividade do sistema? Definida a questão problema é iniciada a busca pelos dados que podem gerar informação para a solução.

Mesmo em visitas técnicas mais demoradas, é possível realizar um diagnóstico da situação atual, mas não isolar e estudar outros níveis dos principais fatores.

Se por um lado as visitas técnicas não são suficientes para estudos mais aprofundados, por 
outro representam uma oportunidade sem igual para a coleta de dados relacionados aos tempos de processamento, atributos das peças produzidas, uso de recursos e capacidades de armazenamento. Isso pode ser destacado como mais um ganho da aplicação da metodologia, pois logo no primeiro semestre de implantação foi observada a valorização das visitas técnicas, que passaram a ter um propósito definido, passaram a fazer parte de uma atividade de mais longo prazo, em suma, mais desafiadora. As visitas técnicas passaram a ser também uma oportunidade para coleta das informações necessárias para a elaboração do modelo conceitual e computacional.

$\mathrm{Na}$ etapa de coleta de dados é realizada uma importante vinculação dessa atividade com a disciplina de Estatística. São reforçados conceitos vinculados à amostragem, como a relação entre o tamanho da amostra e a confiabilidade dos dados, a necessidade de observação da representatividade, independência e aleatoriedades das observações, além da variação que deverá ser considerada no modelo computacional.

Normalmente são coletados dados de tempos de processamento por cada estação de trabalho e de atributos que levam à tomada das decisões previstas no Fluxograma. Para o exemplo proposto, foram tomados dados de diâmetro das toras e tempos de processamento em cada máquina. A coleta pode ser feita por meio de medição direta dos tempos e atributos ou por meio de sensores e filmagens. Esses últimos recursos permitem aumentar consideravelmente o tamanho das amostras sem demandar longos tempos de coleta de dados.

A entrada dos dados obtidos no sistema real em um modelo computacional pressupõe o ajuste a um modelo de probabilidade e se caracteriza como atividade que finaliza o modelo conceitual e inicia o modelo computacional.

$\mathrm{Na}$ experiência que está sendo aqui relatada, o ajuste é realizado com o pacote fitdistrplus (DELIGNETTE-MULLER; DUTANG, 2015) em linguagem aberta $R$ ( $R$ CORE TEAM, 2020). Modelos probabilísticos, juntamente com as estimativas dos parâmetros, são os elementos que permitem contemplar as aleatoriedades no modelo computacional.

A verificação do modelo passa pela aplicação de ferramentas estatísticas como os testes de aderência (Qui-quadrado e/ou Kolmogorov-Smirnov), testes de comparação de médias, estabelecimento de intervalos de confiança, entre outras, com o objetivo de verificar se o software representa com fidelidade os dados obtidos no sistema real (SARGENT, 2013). As representações gráficas obtidas a partir dos testes estatísticos realizados permitem observar que os dados obtidos pela simulação se concentram de maneira semelhante aos valores observados.

O modelo computacional, finalmente, permitirá que seja respondida a pergunta colocada, uma vez que tem como saída a taxa de utilização de cada máquina e o número de peças produzidas como resultado dos diferentes diâmetros das toras na entrada do sistema. A determinação do efeito do fator diâmetro das toras foi exemplificada, sem necessidade de alteração na operação da serraria.

\section{Indissociação entre ensino, pesquisa e extensão como fator crítico de sucesso}

Antes da adoção da metodologia SPMMS nas disciplinas que envolvem processos de industrialização da madeira, dois projetos de iniciação científica sequenciais foram desenvolvidos. O objetivo dessa integração pesquisa-ensino foi observar como alunos de graduação, com mais tempo disponível, aceitam a ferramenta; antecipar as dificuldades ao longo do processo; e formar alunos bolsistas que pudessem dar suporte (peer-instruction) aos alunos das disciplinas quando a ferramenta fosse implantada em sala. $\mathrm{O}$ entendimento do sistema de produção, que era um pátio de toras, com as operações de recebimento, quantificação, descarregamento, classificação, armazenamento e entrega, era simples para os alunos. Entretanto, ficou claro que a dificuldade estaria vinculada ao uso do software e como determinadas decisões deveriam ser implementadas. Na época era utilizado um software comercial na versão estudantil, com suporte limitado pelo fabricante. 
Após dois anos de observação na iniciação científica, decidiu-se adotar a ferramenta nas disciplinas Tecnologia de Produtos Florestais II, especialmente na unidade que envolve os processos de serraria; Tecnologia de Produtos Florestais III, especialmente na unidade que aborda a produção de portas e compensados; Estágio Supervisionado em Engenharia Florestal; e Trabalho de Conclusão do Curso.

Com a chegada dos alunos que experimentaram a modelagem e simulação como ferramenta de ensino no mestrado, outra possibilidade de integração entre diferentes níveis de ensino se estabeleceu. A escolha de um tema que envolve modelagem e simulação por uma aluna de mestrado permitiu o aprofundamento da análise de processos de industrialização. $\mathrm{O}$ trabalho foi desenvolvido em parceria com uma empresa líder na região, constituindo-se uma atividade de extensão. Parte desse conhecimento foi transferido pela mestranda aos alunos de graduação na disciplina Estágio Docente. A Figura 1 procura demonstrar a sinergia promovida entre os principais componentes da universidade ensino em diferentes níveis, pesquisa e extensão - no desenvolvimento da metodologia apresentada.

Figura 1 - Principais elementos envolvidos na implantação da modelagem e simulação de eventos discretos como ferramenta de aprendizagem ativa de processos de industrialização da madeira

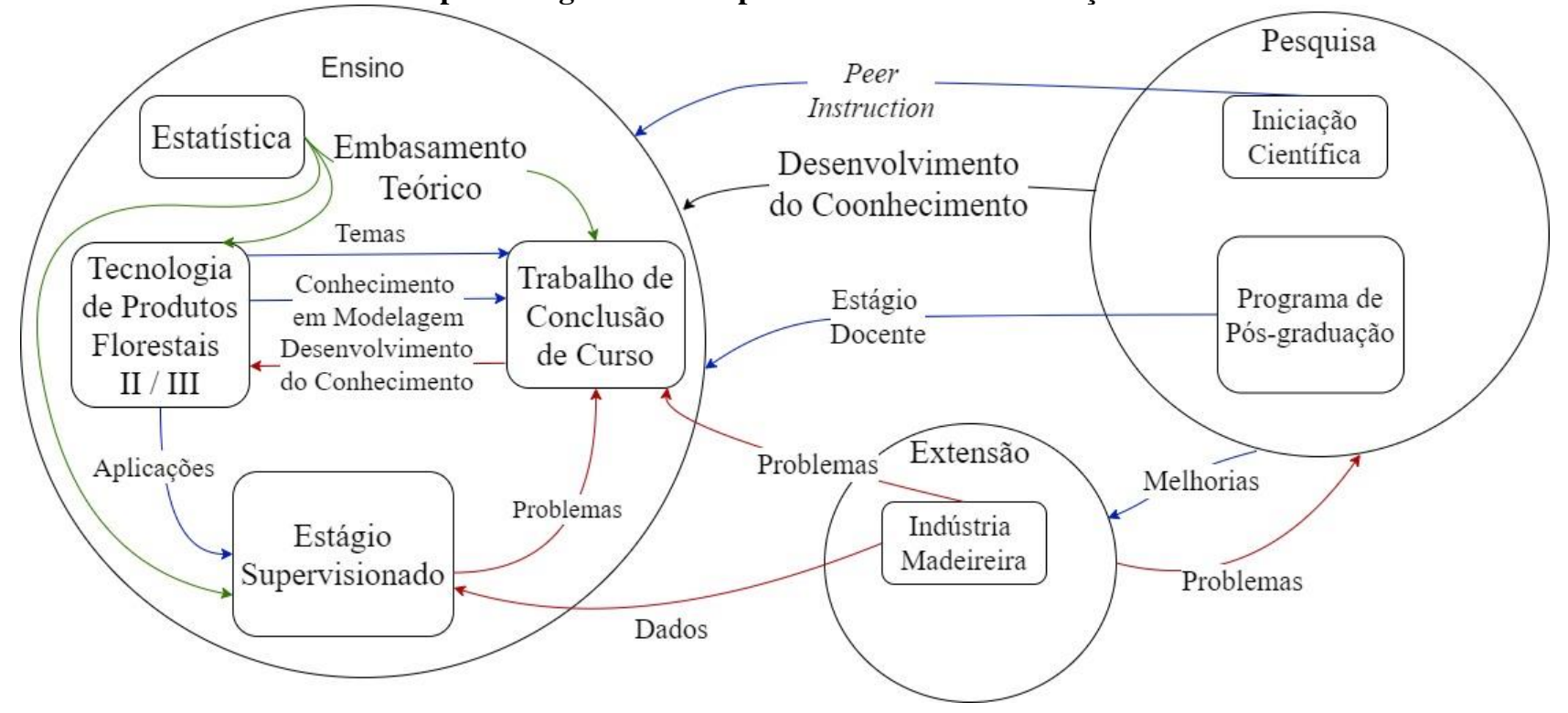

Fonte: acervo dos autores.

Em síntese, os resultados principais foram uma maior e melhor integração com outras disciplinas, especialmente de Estatística; integração entre diferentes níveis de ensino; valorização das visitas técnicas em unidades industriais, ampliando o conceito de sala de aula; melhora da capacidade de abstração; mudança de foco da aprendizagem através da audiência ao professor pela aprendizagem pela modelagem; redução da dependência e submissão ao professor.

\section{CONCLUSÕES}

A modelagem conceitual, computacional e a simulação de eventos discretos, seguindo a metodologia SPMMS apresentada neste trabalho, mostrou-se ferramentas úteis para complementação da formação de engenheiros. Em especial no caso da Engenharia Florestal, entre os ganhos observados destacam-se: a interdisciplinaridade; o aumento do senso de responsabilidade como agente ativo da aprendizagem; e $\mathrm{o}$ fortalecimento das competências e habilidades previstas nas 
diretrizes curriculares nacionais para engenharia florestal.

\section{REFERÊNCIAS}

BABULAK, E.; WANG, M. Discrete Event Simulation: state of the art. Discrete Event Simulations, [S.L.], p. 1-13, 18 ago. 2010. Sciyo.

BACICH, L.; MORAN, J. (orgs.) Metodologias ativas para uma educação inovadora: uma abordagem teórico-prática. Porto Alegre: Penso, 2018.

CNE/CES. Conselho Nacional de Educação/Câmara de Educação Superior. Resolução no 3, de 2 de fevereiro de 2006. Institui as Diretrizes Curriculares Nacionais para o curso de graduação em Engenharia Florestal e dá outras providências. Diário Oficial da União, de 03/02/2006, Seção I, pág. 33-34.

CHWIF, L.; MEDINA, A. C. Modelagem e simulação de eventos discretos: teoria \& aplicações. 4. ed. São Paulo: Elsevier, 2015.

DELIGNETTE-MULLER, M. L.; DUTANG, C.. Fitdistrplus: anrpackage for fitting distributions. Journal of Statistical Software, [S.L.], v. 64, n. 4, p. 1-34, 2015.

ELMÔR FILHO, G.; SAUER, L. Z.; ALMEIDA, N. N.; VILLAS-BOAS, V. Uma nova sala de aula é possível. Rio de Janeiro: LTC, 2019.

FURB. Universidade Regional de Blumenau. Projeto pedagógico do curso de engenharia florestal. Blumenau: Furb, 2010. 112 p. Disponível

em:http://www.furb.br/web/upl/graduacao/pro jeto_pedagogico/201608081639020.PPCENG .FLORESTAL 2011.pdf. Acesso em: 25 nov. 2020.

HERNÁNDEZ-DE-MENÉNDEZ, M. et al. Active learning in engineering education. A review of fundamentals, best practices and experiences. International Journal on Interactive Design and Manufacturing (Ijidem), [S.L.], v. 13, n. 3, p. 909-922, 13 fev. 2019.
LÓPEZ-QUEROL, S. et al. Improving Civil Engineering Education: transportation geotechnics taught through project-based learning methodologies. Journal of Professional Issues In Engineering Education And Practice, [S.L.], v. 141, n. 1, p. 040140071-040140077, jan. 2015.

MESQUITA, M. A.; MARIZ, F. B. A. R.; TOMOTANI, J. V. The Skateboard Factory: a teaching case on discrete-event simulation. Production, [S.L.], v. 27, p. 1-11, 2017.

MOORE, K.; JONES, C.; FRAZIER, R. S. Engineering Education for Generation $\mathrm{Z}$. American Journal of Engineering Education (Ajee), [S.L.], v. 8, n. 2, p. 111-126, $1 \mathrm{dez} .2017$.

R CORE TEAM. R: a language and environment for statistical computing. $\mathrm{r}$ foundation for statistical computing. R Foundation for Statistical Computing. 2020. Disponível em: www.R-project.org. Acesso em: 26 nov. 2020.

RIBEIRO, L. R. C. Aprendizagem Baseada em Problemas (PBL) na educação em engenharia. Revista de Ensino de Engenharia, v. 27, n. 2, p.23-32, 2008.

ROBINSON, S. Conceptual modelling for simulation Part I: definition and requirements. Journal of The Operational Research Society, [S.L.], v. 59, n. 3, p. 278-290, mar., 2008a.

ROBINSON, S. Conceptual modelling for simulation Part II: a framework for conceptual modelling. Journal of The Operational Research Society, [S.L.], v. 59, n. 3, p. 291304, mar., 2008b.

ROBINSON, S. Conceptual modelling for simulation: progress and grand challenges. Journal of Simulation, [S.L.], v. 14, n. 1, p. 120, 2020.

ROMEU, J. L. On Simulation and Statistical Education. American Journal of Mathematical and Management Sciences, [S.L.], v. 17, n. 3-4, p. 397-420, fev. 1997.

SANTAELLA, L. Comunicação ubíqua: repercussões na cultura e na educação. São Paulo: Paulus, 2013. 
SARGENT, R. G. Verification and validation of simulation models. Journal of Simulation, [S.L.], v. 7, n. 1, p. 12-24, fev. 2013.

SPEDDING, T.A.; SUN, G.Q. Application of discrete event simulation to the activity-based costing of manufacturing systems. International Journal of Production Economics, [S.L.], v. 58, n. 3, p. 289-301, jan. 1999.

STRIMEL, G. J.; GRUBBS, M. E.; WELLS, J. G. Engineering Education: A Clear Decision. Technology and Engineering Teacher, v. 76, n. 4, p. 18-24, jan. 2017.
TAO, C.; ZHANG, J.; CHENG, B.; LIU, Y. An Assessment of the Impact of Spatial Agglomeration on the Quality of China's Wood Processing Industry Products. Sustainability, [S.L.], v. 11 , n. 14 , p. 39613978, 21 jul. 2019.

ZEE, D-J. D et al. Education on conceptual modeling for simulation: challenging the art. In: WINTER SIMULATION CONFERENCE, 2010., 2010, Baltimore. Proceedings... Baltimore: Ieee Press, 2010. p. 290-304. Disponível em: https://doi.org/10.1109/wsc.2010.5679156. Acesso em: 25 nov. 2020.

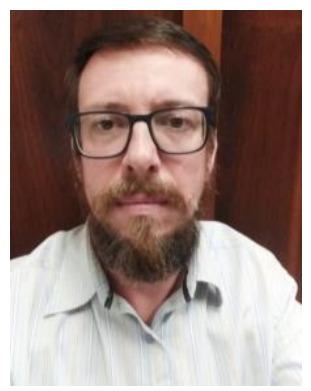

\section{DADOS BIOGRÁFICOS DOS AUTORES}

Jackson Roberto Eleotério - Graduado em Engenharia Florestal pela UFSM (1997), com mestrado em Ciência e Tecnologia da Madeira pela ESALQ/USP (2000) e doutorado em Engenharia Química pela UFSC (2009). Professor da Universidade Regional de Blumenau (FURB) desde 2000. Atua na área de processos de industrialização da madeira, simulação de processos e engenharia da qualidade.

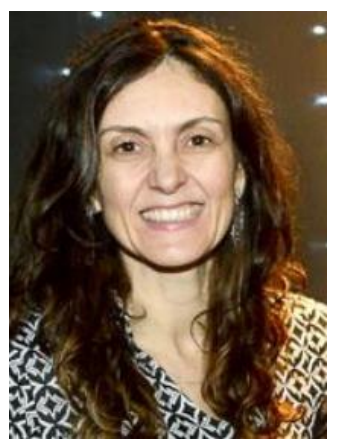

Simone Leal Schwertl - Graduada em Matemática (1991) pela FURB, especialização em Desenho (1996), mestrado em Engenharia de Produção (1999) e doutorado em Educação Científica e Tecnológica (2016) pela UFSC. Professora da Universidade Regional de Blumenau (FURB) desde 2000. Atua como professora da área da Matemática em cursos de engenharia e licenciatura em Matemática. 